\title{
Heimlich Maneuver Complications: A Systematic Review
}

\author{
(D) Mohsen Ebrahimi ${ }^{1}$, (D) Amir Mirhaghi² \\ 1Department of Emergency Medicine, Faculty of Medicine, Mashhad University of Medical Sciences, Mashhad, Iran \\ 2Nursing and Midwifery Care Research Center, Mashhad University of Medical Sciences, Mashhad, Iran
}

\begin{abstract}
Aim: Life-threatening complications have been reported due to the widespread use of the Heimlich maneuver. As the extent of associated injuries has not been well established, a systematic review of the complications of the Heimlich maneuver was conducted.

Materials and Methods: Studies were identified through literature search in MEDLINE, Web of Science and SCOPUS up to August, 2018 with keywords related to "Heimlich maneuver" and "Abdominal thrust". The inclusion criteria were defined as case reports reporting complications due to the Heimlich maneuver and case reports with documented injuries. Original studies, reviews, conference proceedings, commentaries, and case reports with incomplete data were excluded. The CARE (CAse REport) guideline was used to assess the quality of case reports

Results: Forty-eight eligible studies involving 51 cases were included. Patient median age was 62 years and 35\% of them were female. Dyspnea and abdominal pain were the most common symptoms. Gastric rupture was more likely to be associated with hospital admission, but mortality was most associated with aorta injury. Twenty-five percent of cases with organ damage survived.

Conclusion: According to case reports, the Heimlich maneuver is associated with serious complications especially in elderly patients. Life threatening injuries associated with the Heimlich maneuver suggest that this procedure should be substituted with a safer procedure such as chest thrusts or chest compressions. Investigation of an alternative procedure to remove foreign body airway obstruction is recommended in further studies.
\end{abstract}

Keywords: Heimlich maneuver, abdominal thrust, chocking, first aid

\section{Introduction}

The Heimlich maneuver was introduced in 1974 in order to prevent death from food asphyxiation (1). It is clear that any person informed on the procedure can perform the maneuver by not needing any special instrument. The elderly as a vulnerable population are predominantly affected by the Heimlich maneuver (2). Elderly patients are highly susceptible to choking due to neuromuscular disorders such as age-related changes of the nervous system, muscular dystrophy and dental problems which put them into a riskier situation during swallowing. Defective laryngeal closure, failure of bolus containment, transitional phase dissociation, and incomplete bolus transport are the main oropharyngeal abnormalities that are associated with choking episode (3). The elderly people may be even accompanied by frailty and comorbidity (2). Therefore, elderly patients received Heimlich maneuver more commonly than any other populations. Soon after, "a quick upward thrust" (abdominal thrust) became the weakest link due to excessive force directed to internal organs especially in the elderly. Although the American Medical Association endorsed the Heimlich maneuver in 1975 (4), the aggressive nature of the Heimlich maneuver raised concern about the safety measures among academic community. Despite the widespread use due to the fact that it was simple to learn and effective to save lives, academic community remained suspicious (5). They conducted research on choking animals and even humans to examine the underlying mechanism (6-8), and also published case reports of serious complications. One year later, a case of 
ruptured stomach was the first documented life threatening complication. Since then,complications of the Heimlich maneuver have been reported in a consistent manner each year up to now (9). Injuries to the stomach, intestine, pancreas, aorta, esophagus and ribs were reported in the literature $(2,10)$. Despite the poor generalizability and weak cause-effect relationship, case reports have a unique ability to be novel and hypothetic as well as explanatory (11). The educational merit of case report is also remarkable. Growing evidence of serious complications suggests that there is a genuine need to perform a systematic review on the case reports of the Heimlich maneuver. In addition, the Heimlich maneuver is mainly supported by editorial and commentaries and also it suffers from lack of evidence, so case reports are the most reliable evidence in this situation. The study aimed to systematically review the case reports on the Heimlich maneuver complications.

\section{Materials and Methods}

MEDLINE, Web of Science, and Scopus were searched beginning from their starting date to August, 2018. The search keywords were detailed asHeimlich maneuver, Heimlich manoeuvreand abdominal thrust. Duplicated citations were removed. Abstracts were initially screened to reveal relevant case reports. The inclusion criteria were defined as (1) case reports reporting complications due to the Heimlich maneuver and (2) case reports with documented injuries. Original studies, reviews, conference proceedings, commentaries, and case reports with incomplete data were excluded. All abstracts published in English were retrieved. The citation lists of included case reports were screened to discover additional case reports which might have been missed in primary search. Search strategy was illustrated in Figure 1.

Data including first author, year of publication, sex, age, event, chief complaint, type of the Heimlich maneuver intervention, role of caregiver who performed the Heimlich maneuver, main complications, comorbidity, treatment plan, and mortality status were collected. Two independent researchers (A.M. and M.E.) reviewed studies. Disagreements were resolved by consensus. The CARE (CAse REport) guideline was used to assess the quality of case reports (12). Systematic data collection according to the CARE guideline provides evidence to evaluate case reports which have been published in medical literature.

\section{Results}

Two hundred potential studies through Medline, 205 through Web of Science and 331 through Scopus were identified. Three hundred and twenty-eight of them were excluded due to duplication. Initial screening excluded 317 abstracts because they were unrelated, original research, commentary and review studies. As a result, full-text analyses of 91 remaining studies were performed. Secondary screening excluded 47 articles. Four articles were retrieved from reference list. The final inclusion covered 48 articles (Figure 1). The characteristics of all included studies are summarized in Appendix A. All relevant case reports met minimum requirements to be included in regard to the quality assessment.

The median age of the patients was 62 years and it ranged from 3 to 93 years and $31 \%$ were older than 75 years. Thirty-five percent of patients were female. The most common chief complaints were dyspnea and abdominal pain. Patients had generally received the Heimlich maneuver from bystanders rather than health care providers. Gastric rupture was more likely to be associated with hospital admission, but mortality was most associated with aorta injury. Forty-one percent of cases suffered from comorbidities. Forty percent of cases underwent surgery to repair injuries. Twenty-five percent of cases who had organ damage survived. The details of injures are presented in Appendix A. A summary of injuries associated with the Heimlich maneuver is detailed in Table 1.

\section{Discussion}

The most common injury was gastric rupture, which is reported by academic community. The lesser curvature of the stomach is ruptured in most cases. Weak abdominal muscles, especially

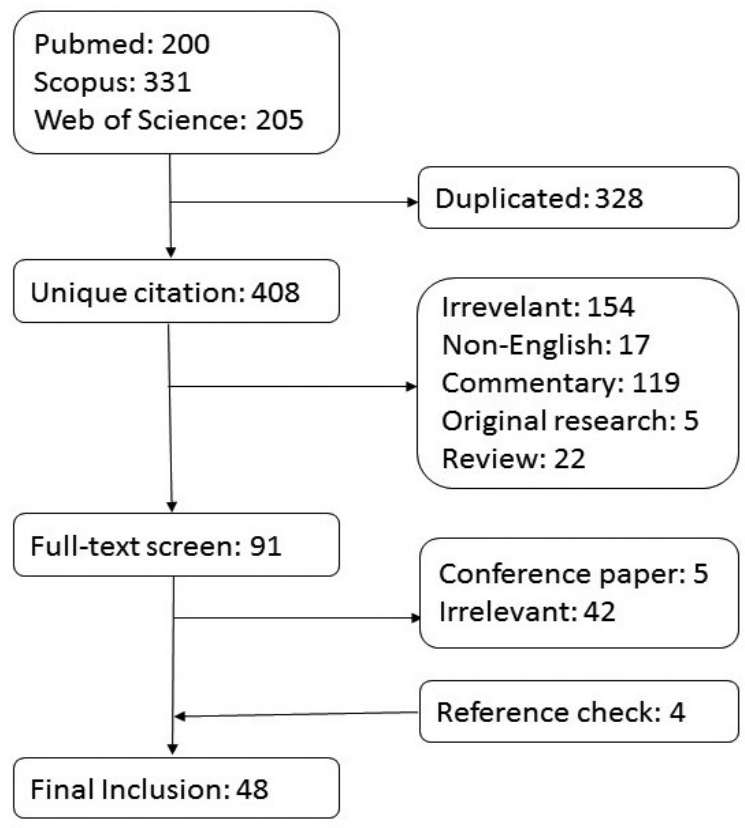

Figure 1. Flowchart of the litarature search and exclusion process 
Table 1. Summary of reported injuries associated with Heimlich maneuver

\begin{tabular}{|c|c|c|c|c|c|c|c|}
\hline Organs & $\begin{array}{l}\text { Studies } \\
\text { (Sample } \\
\text { size) }\end{array}$ & Gender & Age & Common symptoms & Complication & Treatment & Mortality \\
\hline \multicolumn{8}{|l|}{ Lung } \\
\hline Edema $7,12,24,43,44,47^{*}$ & $5(6)$ & $1 \mathrm{~F}: 5 \mathrm{M}$ & $13(5-50)$ & $\begin{array}{l}\text { Dyspnea; emesis; altered } \\
\text { mental status }\end{array}$ & $\begin{array}{l}\text { Post-obstructive } \\
\text { pulmonary edema; } \\
\text { diffuse patchy lobular } \\
\text { airspace disease }\end{array}$ & $\begin{array}{l}\text { Supportive } \\
\text { care }\end{array}$ & 0 \\
\hline $\begin{array}{l}\text { Pneumomediastinum } \\
1,14,22\end{array}$ & $3(3)$ & $3 \mathrm{M}$ & $3,19,39$ & $\begin{array}{l}\text { Sore throat; dyspnea; } \\
\text { collapsed }\end{array}$ & Pneumomediastinum & $\begin{array}{l}\text { Surgery; } \\
\text { CPR }\end{array}$ & 1 \\
\hline Emphysema 38 & $1(1)$ & $1 \mathrm{M}$ & 56 & Dyspnea & $\begin{array}{l}\text { Emphysematous bulla in } \\
\text { apical region }\end{array}$ & Surgery & 0 \\
\hline \multicolumn{8}{|l|}{ Ribs } \\
\hline Fracture $16,18,30$ & $3(3)$ & $3 \mathrm{~F}$ & $72,79,90$ & Chest pain; pleuritic pain & Rib fractures & Supportive & 0 \\
\hline \multicolumn{8}{|l|}{ Diaphragm } \\
\hline Rupture 26,48 & $2(2)$ & $2 \mathrm{~F}$ & 85,85 & Dyspnea & Hiatal hernia & Surgery & 0 \\
\hline \multicolumn{8}{|l|}{ Esophagus } \\
\hline 6 & $1(1)$ & $1 \mathrm{~F}$ & 45 & $\begin{array}{l}\text { Subcutaneous } \\
\text { emphysema }\end{array}$ & $\begin{array}{l}\text { Pharyngoesophageal } \\
\text { perforation }\end{array}$ & Surgery & 0 \\
\hline $25,28,37,41$ & $4(4)$ & $2 \mathrm{~F}: 2 \mathrm{M}$ & $\begin{array}{l}16,56 \\
61,62\end{array}$ & $\begin{array}{l}\text { Odynophagia; dyspnea } \\
\text { and chest pain }\end{array}$ & $\begin{array}{l}\text { Perforation of the } \\
\text { esophagus }\end{array}$ & Surgery & 0 \\
\hline \multicolumn{8}{|l|}{ Stomach } \\
\hline $\begin{array}{l}\text { Lesser curve } \\
4,5,9,13,17,19,23,34,50,51\end{array}$ & $9(10)$ & $4 \mathrm{~F}: 3 \mathrm{M}$ & $74(57-93)$ & Abdominal pain & $\begin{array}{l}\text { Gastric rupture along the } \\
\text { lesser curvature of the } \\
\text { stomach }\end{array}$ & Surgery & 3 \\
\hline Volvulus 36 & $1(1)$ & $1 \mathrm{~F}$ & 10 & Abdominal pain & $\begin{array}{l}\text { Mesenteroaxial gastric } \\
\text { volvulus }\end{array}$ & Surgery & 0 \\
\hline \multicolumn{8}{|l|}{ Pancreas } \\
\hline 20,29 & $2(2)$ & $2 \mathrm{M}$ & 3,11 & Abdominal pain & Transection; cystic mass & Surgery & 0 \\
\hline \multicolumn{8}{|l|}{ Liver } \\
\hline 39,46 & $2(2)$ & $2 \mathrm{M}$ & 84,88 & Abdominal pain & $\begin{array}{l}\text { Laceration in the hepatic } \\
\text { lobe }\end{array}$ & Surgery & 0 \\
\hline \multicolumn{8}{|l|}{ Small intestine } \\
\hline 42 & $1(1)$ & $1 \mathrm{M}$ & 22 & Vomiting & Jejunum perforation & Surgery & 0 \\
\hline \multicolumn{8}{|l|}{ Spleen } \\
\hline 8 & $1(1)$ & $1 \mathrm{M}$ & 83 & Unconscious & Laceration of spleen & CPR & 1 \\
\hline \multicolumn{8}{|l|}{ Aorta } \\
\hline $\begin{array}{l}\text { Thrombosis } \\
2,27,32,33,35,35\end{array}$ & $5(6)$ & $2 \mathrm{~F}: 4 \mathrm{M}$ & $80(69-84)$ & Paraplegia & $\begin{array}{l}\text { Thrombosis and } \\
\text { occlusion in aorta }\end{array}$ & Surgery & 5 \\
\hline $\begin{array}{l}\text { Tear } \\
15,21,49\end{array}$ & $3(3)$ & $2 \mathrm{~F}: 1 \mathrm{M}$ & $61,76,78$ & $\begin{array}{l}\text { Dyspnea; unconscious; } \\
\text { abdominal pain }\end{array}$ & Dissection of aortic wall & Surgery & 3 \\
\hline Valve 10,40 & $2(2)$ & $1 \mathrm{~F}: 1 \mathrm{M}$ & 74,86 & Dyspnea & $\begin{array}{l}\text { Acute aortic } \\
\text { regurgitation }\end{array}$ & $\begin{array}{l}\text { Surgery; } \\
\text { supportive }\end{array}$ & 0 \\
\hline Stent displacement 31 & $1(1)$ & $1 \mathrm{M}$ & 63 & Abdominal discomfort & $\begin{array}{l}\text { Proximal type } 1 \\
\text { endoleak }\end{array}$ & Surgery & 0 \\
\hline \multicolumn{8}{|l|}{ Vertebra } \\
\hline 11 & $1(1)$ & $1 \mathrm{~F}$ & 80 & Back pain & $\begin{array}{l}\text { Acute compression } \\
\text { deformities at L1-2 levels }\end{array}$ & Kyphoplasty & 0 \\
\hline \multicolumn{8}{|l|}{ Shoulder } \\
\hline 3 & $1(1)$ & $1 \mathrm{M}$ & 48 & Shoulder pain & Rotator cuff tear & Surgery & 0 \\
\hline
\end{tabular}


in the elderly patients cannot protect internal organs compared to those in adults. It is expected that the lesser curvature of the stomach is the most common site of involvement because it directly receives the force generated by abdominal thrust. Upward thrust above the novel generates excessive force toward the stomach. Heimlich stated that "place your fist (thumb side against the victim's abdomen) slightly above the novel and below the rib cage and grasp your fist with your other hand and press into the victim's abdomen with a quick upward thrust.", so there is a great possibility of the lesser curvature injury especially when the stomach is distended after eating a meal (1). The missing link is the fact that no instruction about how much force is required to dislodge the foreign body is given. An adult is able to produce an excessive force through an abdominal thrust which is unbearable for a senior adult or a child, resulting in fatal internal injury. A hypothesis that a choking person may benefit most from the Heimlich maneuver which is adjusted for a person at the same age may arise. There is a necessity to address this issue in further studies.

It is argued that the Heimlich maneuver may not always be the procedure of choice in all situations (13). For example, a large piece of meat can likely be dislodged by the Heimlich maneuver than more viscous materials such as peanut butter. In such situation, finger sweep technique may be more effective than the Heimlich maneuver to resolve choking especially in children. The Heimlich maneuver may not be the procedure of choice in case of esophageal impaction either. Esophageal impaction may happen in individuals who are able to vocalize during the choking. There is great possibility that untrained people may not distinguish a pseudo-choking state from an airway obstruction episode (14). In such situation, the application of abdominal thrust causes an increase in intraluminal pressures in internal organs. Impacted food in esophagus deteriorates the situation because it does not allow the pressure to be diminished and weak structure of esophagus escalates injury to a life threatening condition (4). Many scholars argued that untrained rescuers may be a part of this puzzle.

This review shows that patients may suffer from serious injuries in spite of professional help received from trained people such as nurses. Razaboni et al. (15) reported a case of jejunum perforation, who received abdominal thrust from a trained rescuer. It implies that excessive force may play a greater role than proper technique in developing injuries. The risk is much higher in choking persons with comorbidities, suggesting that individual characteristics are influential indeed. In addition, experimental studies have indicated that the intrathoracic pressure induced by upward abdominal thrust does not differ from backward abdominal thrust significantly; implying that rescuers may not require to perform the Heimlich maneuver in upward direction necessarily (16). In this way, Roehm et al. (17) argued that abdominal thrust without any upward motion may result in an increased risk of trauma to the abdominal aorta. Therefore, it can be concluded that excessive force apart from upward or backward direction is strongly associated with internal organ damage.

Alternative maneuvers: WhileAmerican Red Cross and European Resuscitation Council endorse abdominal thrusts to manage foreign body airway obstruction (FBAO) (18), the Australian and New Zealand Committee on Resuscitation (ANZCOR) does not recommend abdominal thrusts in the management of FBAO due to life-threatening complications (ANZCOR) (19). Instead, back blows and chest thrusts have been endorsed by ANZCOR. Apart from life threatening complications associated with the Heimlich maneuver, literature suggests that chest compression may produce more peak airway pressure than the Heimlich maneuver. Langhelle et al. (6) indicated that chest compression was more effective than the Heimlich maneuver in managing FBAO in unconscious patients. They showed that peak airway pressure was significantly lower with abdominal thrusts compared to chest compressions ( $26.4 \pm 19.8$ versus $40.8 \pm 16.4$ $\mathrm{CmH}_{2}{ }^{\circ}$ ) among 12 unselected cadavers. In this way, Guildner et al. (20) concluded both the chest thrusts produced significantly better results than did the abdominal thrust on six adult male anesthetized volunteers and introduced chest thrust as technique of choice. Therefore, it raises concern about widespread interest and popularity of the Heimlich maneuver. In fact, experiments show that chest thrusts are more effective than abdominal thrust in terms of generating higher peak airway pressure.

In addition, several studies have been performed on airway peak pressure in different positions to simulate choking episode. Pavitt et al. (16) indicated that self-administered thrusts over the back of a chair delivered significantly greater pressure than the Heimlich maneuver did and it might increase the chance of injury, too. A manikin study revealed that the lying down abdominal thrust was associated with higher peak pressures than the standing abdominal thrust (22.6 \pm 2.8 versus $11.5 \pm 2.6)$ (7). However, another study indicated that abdominal thrusts produced greater airway pressure than chest thrusts among pigs (13.8 6 6.7 versus $6.5 \pm 3$ ), the major difference in the chest anatomy between human and pig weakens the generalizability of findings (21,22). Finally, Blain et al. (23) proposed the table maneuver as a safer alternative to the Heimlich maneuver. It is performed by giving sharp blows in a choking person who has been laid down on a table in the prone position with the head facing downwards and the arms hanging over the side of the table. They argued that this maneuver was associated with better results than the Heimlich maneuver. 


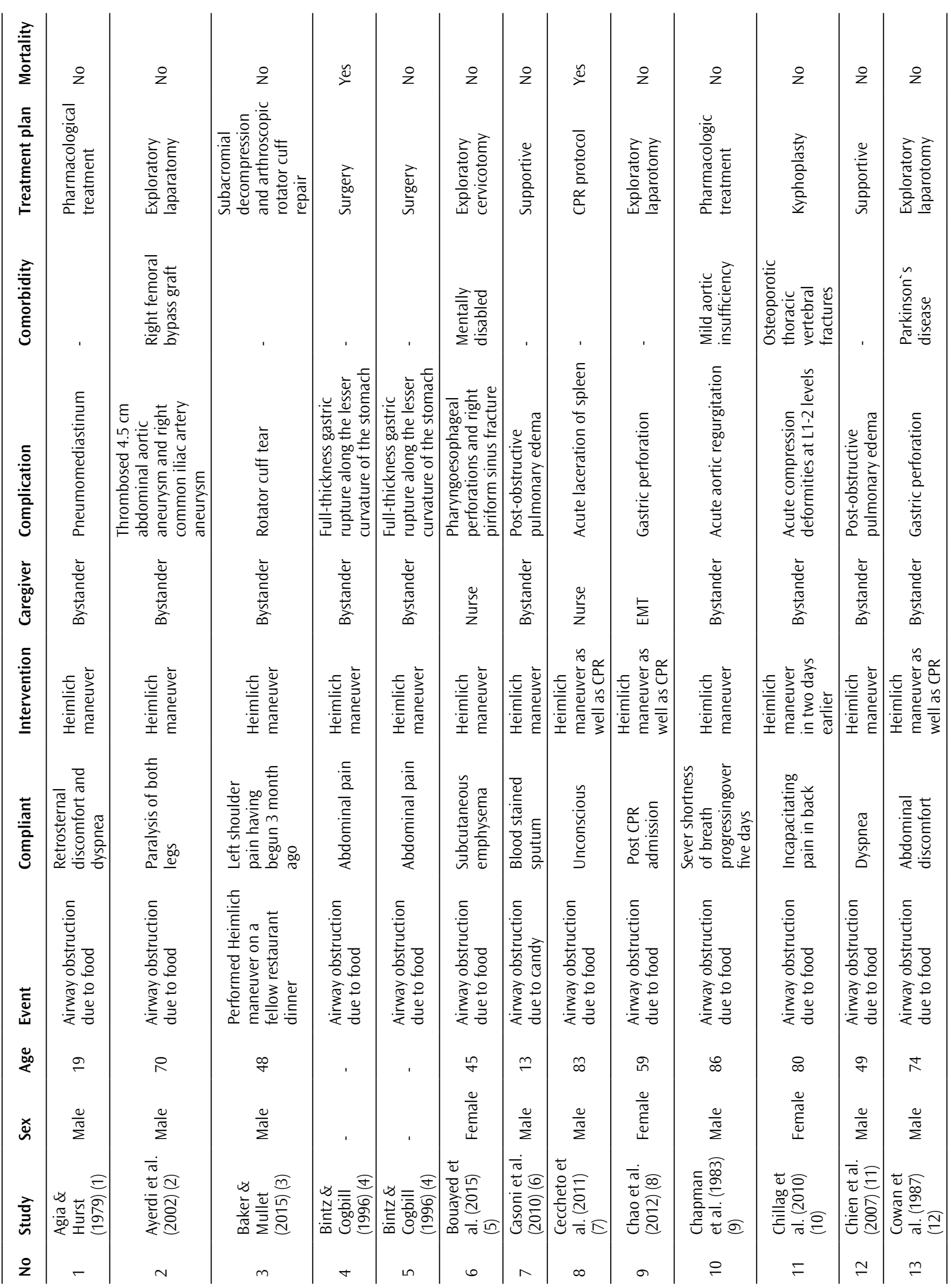




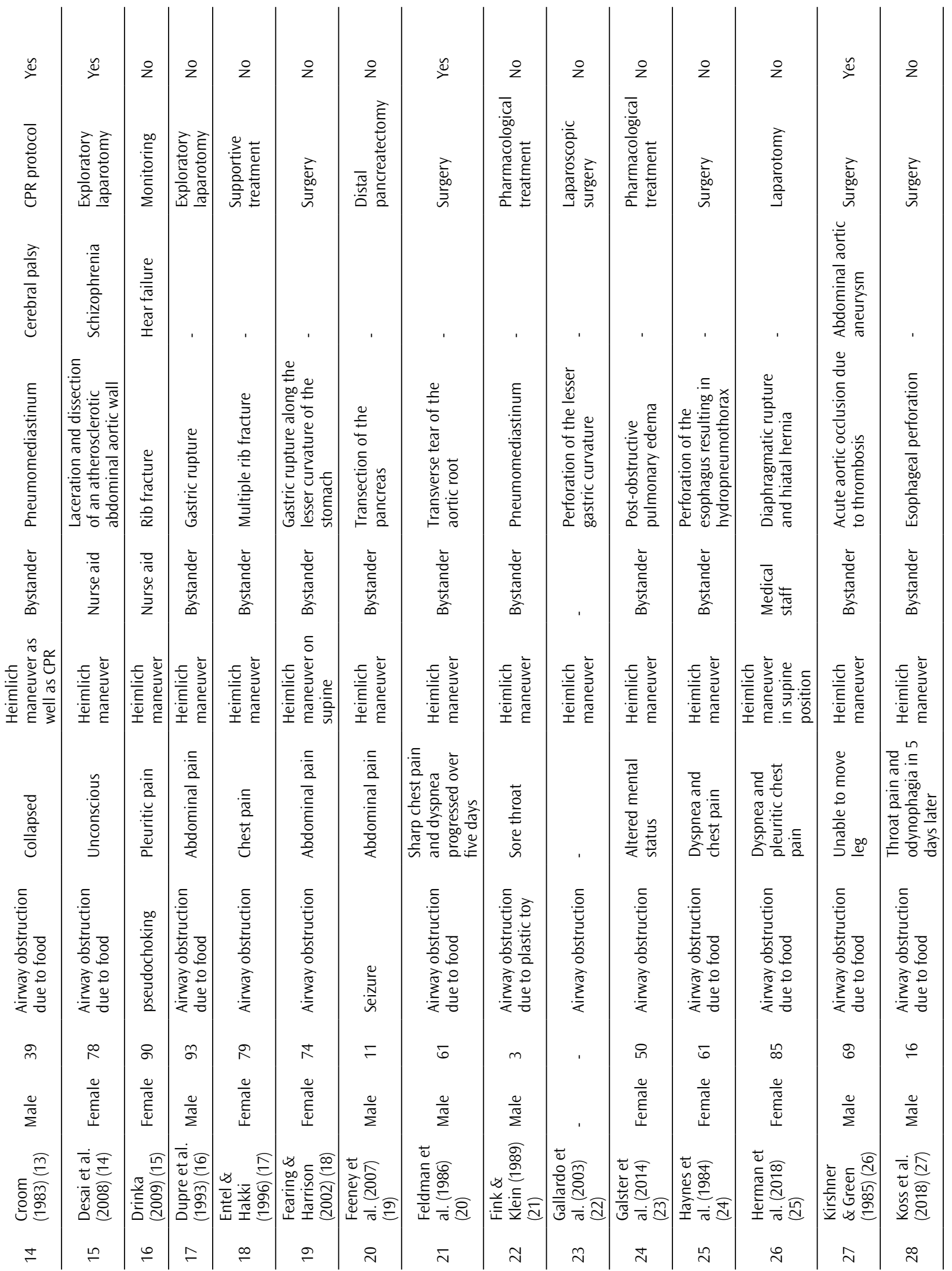




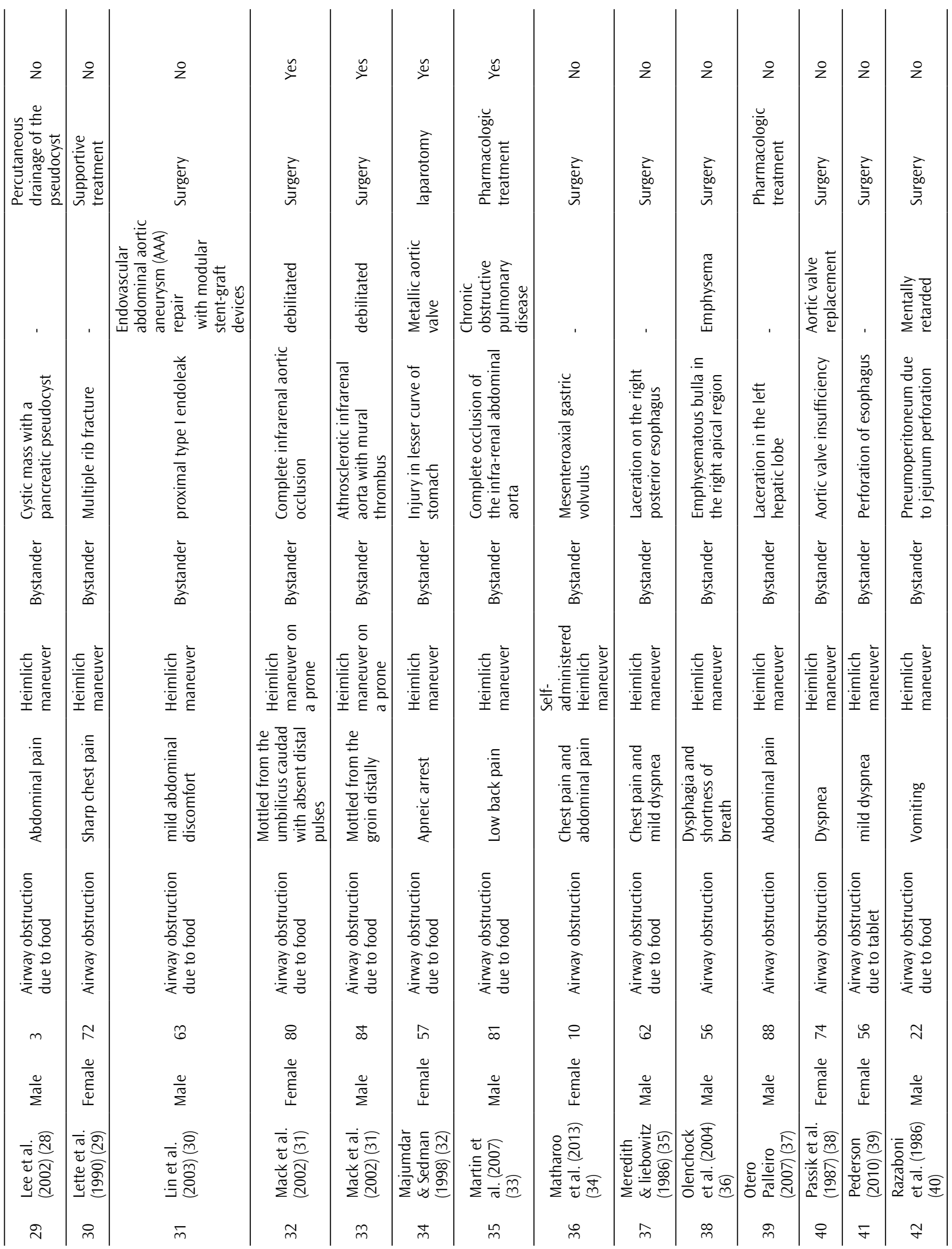




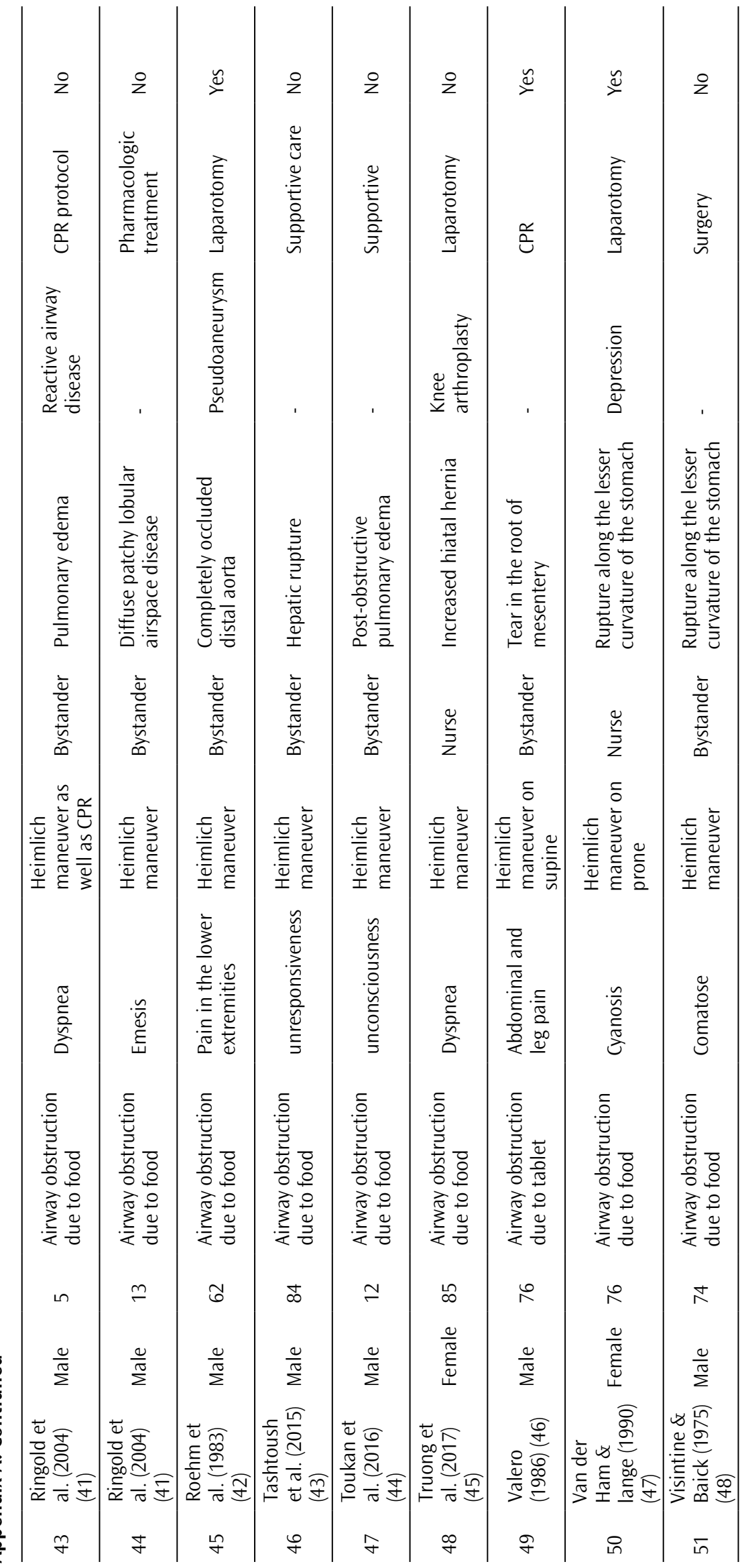

While case reports studies suffer from poor generalizability, systematic review of case series reveals cumulative effect of widespread application of the Heimlich maneuver. It is important because the Heimlich maneuver is mostly supported by editorial and there are a few studies on the efficacy of FBAO removal technique. Medical follow-up as well as alarming signs and symptoms must be considered as a key part of the Heimlich maneuver instruction. In this case, a correct estimation of complications of the Heimlich maneuver can be achieved. The Heimlich maneuver was introduced in 1974. Some journals were not published electronically in the 1970 decade, so there is a probability that some case reports would not be accessible to the electronic search.

\section{Conclusion}

Heimlich maneuver is associated with serious complications especially in elderly patients. Organ damage, especially abdominal aorta injury is the most common fatal injury. Life threatening injuries associated with Heimlich maneuver suggest that this procedure must be substituted with a safer procedure such as chest thrusts or chest compressions. Investigation of an alternative procedure to remove FBAO is recommended in further studies.

\section{Ethics}

Peer-review: Externally and internally peerreviewed.

\section{Authorship Contributions}

Surgical and Medical Practices: N/A.

Concept: A.M., Design: A.M., M.E., Data Collection or Processing: A.M., M.E., Analysis or Interpretation: A.M., M.E., Literature Search: A.M., M.E., Writing: A.M., M.E.

Conflict of Interest: No conflict of interest was declared by the authors.

Financial Disclosure: The authors declared that this study received no financial support. 


\section{References}

1. The Heimlich maneuver. Occupational health nursing. 1976;24:20-1.

2. Chillag S, Krieg J, Bhargava R. The Heimlich maneuver: breaking down the complications. South Med J. 2010;103:147-50.

3. Feinberg MJ, Ekberg 0. Deglutition after near-fatal choking episode: radiologic evaluation. Radiology. 1990;176:637-40.

4. Valero V. Mesenteric laceration complicating a Heimlich maneuver. Ann Emerg Med. 1986;15:105-6.

5. Redding JS. The choking controversy: critique of evidence on the Heimlich maneuver. Critical care medicine. 1979;7:475-9.

6. Langhelle A, Sunde K, Wik L, Steen P. Airway pressure with chest compressions versus Heimlich manoeuvre in recently dead adults with complete airway obstruction. Resuscitation. 2000;44:105-8.

7. Sanuki T, Sugioka S, Son H, Kishimoto N, Kotani J. Comparison of two methods for abdominal thrust: a manikin study. Resuscitation. 2009;80:499-500.

8. Ichikawa M, Oishi S, Mochizuki K, Nitta K, Okamoto K, Imamura H. Influence of body position during Heimlich maneuver to relieve supralaryngeal obstruction: a manikin study. Acute Medicine \& Surgery. 2017;4:418-25.

9. Visintine RE, Baick $\mathrm{CH}$. Ruptured stomach after Heimlich maneuver. JAMA. 1975;234:415

10. Fearing NM, Harrison PB. Complications of the heimlich maneuver: case report and literature review. J Trauma. 2002;53:978-9.

11. Nissen T, Wynn R. The clinical case report: a review of its merits and limitations. BMC Res Notes. 2014;7:264.

12. Gagnier JJ, Kienle G, Altman DG, Moher D, Sox H, Riley D. The CARE guidelines: consensus-based clinical case reporting guideline development. BMC Res Notes. 2013;7:223.
13. Brauner DJ. The Heimlich maneuver: procedure of choice? J Am Geriatr Soc. 1987;35:78.

14. Ekberg 0, Feinberg M. Clinical and demographic data in 75 patients with near-fatal choking episodes. Dysphagia. 1992;7:205-8.

15. Razaboni RM, Brathwaite CE, Dwyer WA, Jr. Ruptured jejunum following Heimlich maneuver. J Emerg Med. 1986;4:95-8.

16. Pavitt MJ, Swanton LL, Hind M, Apps M, Polkey MI, Green M, et al. Choking on a foreign body: a physiological study of the effectiveness of abdominal thrust manoeuvres to increase thoracic pressure. Thorax. 2017;72:576-8.

17. Roehm EF, Twiest MW, Williams RC, Jr. Abdominal aortic thrombosis in association with an attempted Heimlich maneuver. JAMA. 1983;249:1186-7.

18. American Red Cross. Adult First Aid/CPR/AED. 2011.

19. The Australian and New Zealand Committee on Resuscitation (ANZCOR). ANZCOR Guidline 4 - Airway 2016 [Available from: https://resus.org.au/ guidelines/\#.

20. Guildner CW, Williams D, Subitch T. Airway obstructed by foreign material: the Heimlich maneuver. JACEP. 1976;5:675-7.

21. Lippmann J, Taylor DM, Slocombe R, McDonald CF, Walker T, Nolan G. Lateral versus anterior thoracic thrusts in the generation of airway pressure in anaesthetised pigs. Resuscitation. 2013;84:515-9.

22. Rottenberg EM. Could there ever be evidence to support using lateral chest thrusts in humans with the victim lying on their side to clear upper-airway obstruction? Resuscitation. 2013;84:e69.

23. Blain H, Bonnafous M, Grovalet N, Jonquet O, David M. The table maneuver: a procedure used with success in four cases of unconscious choking older subjects. Am J Med. 2010;123:1150.e7-9. 